\title{
Benchmark Datasets for Computational Drug Discovery: Pancreatic Cancer and Cardiovascular Disorders as Case Studies
}

\section{George Tsatsaronis}

Biotechnology Center, Technische Universität Dresden, Dresden, Germany http://www.biotec.tu-dresden.de/

ONLINE: $\quad 25$ Oct 2015

KEYWORDS: Computational Drug Discovery, Drug Repositioning, Pancreatic Cancer, Cardiovascular Disorders.

The current issue of the Biomedical Data Journal (BMDJ)on Computational Drug Discovery focuses on the publication of datasets that will allow researchers to investigate deeper computational techniques for drug design and drug repositioning for pancreatic cancer and cardiovascular disorders. Drug repositioning is the task of finding new targets for old drugs and has been in the spotlight for the past few years. The average cost for launching a new drug into the market is estimated to 1.8 billion dollars. Apart from that, the drugs that make it to the market are very few. Notably, from 1999 to 2008, only 50 compounds were FDA approved in the U.S., out of which 17 were identified as arising from target-based discovery methods. This stresses the importance of drug repositioning in the process of drug development, since it accelerates the process, minimizes the associated costs, and, in parallel, contributes to the prevention of noxious adverse events and toxicological liabilities.

Especially for the case studies of pancreatic cancer and cardiovascular disorders, there is a dire need of datasets which would allow researchers to examine drug repositioning opportunities, and certainly could act as an important curating or guiding factor for any future drug design efforts. Given this need, the current issue focuses on datasets in these two cases.

The papers in this issue can be divided in three categories: pancreatic cancer dataset, ${ }^{1}$ general dataset for computational drug repositioning modelling, ${ }^{2}$ and cardiovascular diseases datasets. ${ }^{3,4,5}$

The first paper ${ }^{1}$ presents a dataset from the pancreatic cancer case study. Raw data obtained from exposing the human pancreatic adenocarcinoma cell line Capan-2 to Gemcitabine and the HSP27-inhibitor BVDU ((E)-5-(2-Bromovinyl)-2'-deoxyuridine, RP101) are described. Differential gene expression was displayed using DNA microarrays. The raw measurements of the pairwise comparison of control (untreated), Gemcitabine-, BVDU-, and Gemcitabine with BVDU - treated probes are released.

The second paper ${ }^{2}$ introduces a dataset for computational drug repositioning, comprising 54 drug repositioning cases, the information for which was manually compiled, curated and integrated. The new indications for the reported cases have been approved by FDA or EU RUS in the period 1955-2013. 
The last three papers of this second issue focus on datasets on cardiovascular disorders. Raw data on the association of the HPT (Hypothalamic pituitary-thyroid)-axis and C-reactive protein (CRP) serum concentrations with clinical stroke severity and with functional and cognitive discharge outcomes are presented. ${ }^{3}$ In addition, the expression of the thyroid hormone (TH) deiodinases (D1, D2, D3) and TH receptors (TR $\alpha 1, T R \alpha 2$ and TRB1) in an endothelial microvascular cultured cell model (HMEC-1), after stimulation with triiodothyronine (T3, 10-100nM), thyroxine (T4, 10-100nM), and reverse T3 (rT3,1-10nM) is released as raw data. ${ }^{4}$ Finally, raw data on the association between Type $D$ personalities and coronary artery disease in Lithuanian patients are also released. ${ }^{5}$

In summary, the release of the raw datasets of this issue constitutes perhaps a first important step towards helping researchers by sharing these data openly in examining further potentials for the treatment of pancreatic cancer and cardiovascular disorders.

\section{References}

1 Heinrich J-C, Fahrig R. Differential Gene Expression of Pancreatic Cancer Cell Line Capan-2 in Response to Gemcitabine plus BVDU (RP101) Combinatorial Treatment. Biomed Data J. 2015;1(2):7-9. doi: $10.11610 /$ bmdj.01201.

2 Kissa M, Tsatsaronis G.A Benchmark Dataset for Computational Drug Repositioning. Biomed Data J. 2015;1(2):10-12. doi: 10.11610/bmdj.01202.

3 Bunevicius A, Kazlauskas H, Raskauskiene N, Janusonis V, Bunevicius R. Thyroid Hormone and CReactive Protein Serum Concentrations, Disease Severity and Discharge Outcomes of Ischemic Stroke Patients: A Dataset. Biomed Data J. 2015;1(2):13-18. doi: 10.11610/bmdj.01204.

4 Sabatino L, Balzan S, Lubrano V, lervasi G. Thyroid Hormone Deiodinases and Receptors Are Expressed in Human Endothelial Cells. Biomed Data J. 2015;1(2):19-25. doi: 10.11610/bmdj.01203.

5 Stropute D, Bunevicius A, Staniute M, Brozaitiene J, Bunevicius R. Type D (Distressed) Personality in Lithuanian Patients with Coronary Artery Disease: A Dataset. Biomed Data J. 2015;1(2):26-30. doi: 10.11610/bmdj.01205. 